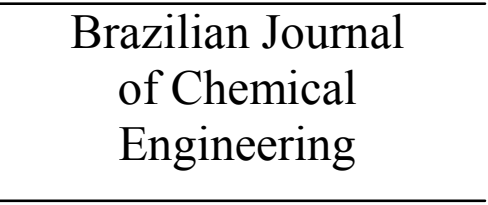

ISSN 0104-6632

Printed in Brazil www.abeq.org.br/bjche

Vol. 23, No. 04, pp. 487 - 496, October - December, 2006

\title{
SOME MODELING AND NUMERICAL ASPECTS OF THE TWO-FLUID SIMULATION OF THE GAS-SOLIDS FLOW IN A CFB RISER
}

\author{
L. Cabezas-Gómez ${ }^{1 *}$, R. C. Silva ${ }^{2}$, and F. E. Milioli ${ }^{1}$ \\ ${ }^{1}$ Núcleo de Engenharia Térmica e Fluidos, Departamento de Engenharia Mecânica, EESC, USP, \\ Av. Trabalhador São-Carlense 400, CEP: 13566-590, São Carlos - SP, Brasil. \\ E-mail: lubencg@sc.usp.br. \\ ${ }^{2}$ Departamento de Ciências Exatas, Campus Três Lagoas, Universidade Federal de Mato Grosso do Sul, \\ Av. Ranulpho Marques Leal 3484, CEP: 79620-080, Três Lagoas - MS, Brasil. \\ E-mail: rcsilva@ceul.ufms.br. \\ E-mail:milioli@sc.usp.br.
}

(Received: April 8, 2005 ; Accepted: July 7, 2006)

\begin{abstract}
The gas-solids flow in a CFB riser is simulated applying two-fluid modeling. Two different procedures are used for the calculation of the solids phase pressure and stress tensor: the traditional procedure and an algebraic version of the kinetic theory of granular flows. Three different numerical meshes and two different discretization schemes for the advective terms are used. Results are compared to available experimental data from the literature. The effects of the solids phase modeling procedure, advection discretization scheme, and mesh size are discussed.

Keywords: Gas-solids flow; Riser; Circulating fluidized bed; Two-fluid model; Kinetic theory of granular flows; MFIX.
\end{abstract}

\section{INTRODUCTION}

In this work results of numerical simulation are presented for the gas-solids two-phase flow in the riser of a circulating fluidized bed (CFB). Two different procedures are considered for the calculation of the solids phase pressure and stress tensor. These are the so-called traditional procedure, which is based on empirical correlations, and an algebraic version of the kinetic theory of granular flows (KTGF), which is based on theoretical correlations. Several studies reported in the literature have applied the above procedures. The traditional procedure was applied, for instance, by Tsuo and
Gidaspow (1990), Sun and Gidaspow (1999), Huilin and Gidaspow (2003), and Cabezas-Gómez and Milioli (2003, 2004, 2005a,b), among others. The procedure based on the algebraic version of the KTGF was applied, for instance, by Syamlal et al. (1993), Boemer et al. (1995), van Wachem et al. (1998), and Guenther and Syamlal (2001), among others.

An algebraic version of the KTGF was applied by Syamlal et al. (1993), offering the possibility of convergence acceleration by directly computing the granular temperature from a simple algebraic equation instead of solving a complex partial differential equation (PDE) for the conservation of

*To whom correspondence should be addressed 
granular energy. Thus, the most notable difference between the algebraic KTGF and other versions of this theory is the use of a simplified balance equation for the calculation of granular temperature. In this procedure both convection and diffusion processes are neglected and only local stationary dissipation of granular energy is taken into account. From the computed granular temperature, and applying theoretical correlations developed by Lun et al. (1984), solids phase pressure and viscosities are determined. According to van Wachem et al. (1998), this procedure is valid only for higher values of the solids volumetric fraction and relatively low values of solids velocity. In such a regime the granular energy is mostly dissipated locally. The approach is clearly more appropriate for gas-solids flows in bubbling fluidized beds. However, in accordance with the literature, the procedure is used in the present work to simulate the gas-solids flow in a CFB riser, showing coherent results.

In the traditional procedure solids phase dynamic viscosity is taken from experiment as a constant, while solids phase pressure is computed as a function of an elasticity modulus accounting for collisional interactions between particles, which is determined from empirical correlations. Recently this procedure was successfully applied by Cabezas-Gómez and Milioli (2003, 2004 and 2005a,b) to simulate the gassolids flow in a CFB riser.

In this work a modification of the algebraic KTGF procedure has also been considered along with the conventional proposition. In this modified version the algebraic KTGF is used for modeling solids phase viscosities, while the solids phase pressure is modeled applying the empirical correlation of the traditional procedure. It is, therefore, a hybrid procedure combining elements of both the traditional and the algebraic KTGF procedures. In fact, the present simulations show that the hybrid modified procedure provides better predictions than the algebraic KTGF procedure.

All the numerical simulations were performed for the CFB installation described in Luo (1987). Besides the three different procedures accounting for solids phase pressure and stress tensor, three different computational meshes and two different interpolation schemes for the advective terms were used.

\section{MATHEMATICAL MODEL}

The simulations in the present work were performed using the MFIX computer model (Syamlal et al., 1993). The code is widely used for multiphase gas-solids flow simulation (Guenter and Syamlal, 2001, Gelderbloom et al., 2003, Gera et al., 2004, among others). The mathematical models used are presented in Tables 1 and 2. In Table 1 the traditional model is presented, while in Table 2 the algebraic KTGF and the hybrid modified models are presented.

In Tables 1 and 2, the subscripts (g) and (s) stand for gas and solids phases, respectively. $\mathrm{v}_{\mathrm{g}}$ and $\mathrm{v}_{\mathrm{s}}$ are local temporal velocities $(\mathrm{m} / \mathrm{s}), \rho_{\mathrm{g}}$ and $\rho_{\mathrm{s}}$ are densities $\left(\mathrm{kg} / \mathrm{m}^{3}\right), \alpha_{\mathrm{g}}$ and $\alpha_{\mathrm{s}}$ represent volumetric fractions, and $\tau_{\mathrm{g}}$ and $\tau_{\mathrm{s}}$ account for viscous stress tensors $(\mathrm{Pa})$. Also, $\mathrm{P}$ is the thermodynamic gas pressure $(\mathrm{Pa}), \mathrm{g}$ is the gravity acceleration $\left(\mathrm{m} / \mathrm{s}^{2}\right), \mathrm{g}_{0}$ stands for the radial distribution function, $G$ is the solids-phase elasticity modulus $\left(\mathrm{N} / \mathrm{m}^{2}\right)$, and $\beta$ is the interface drag function $\left(\mathrm{kg} / \mathrm{m}^{2}-\mathrm{s}\right) . \mathrm{C}_{\mathrm{Ds}}$ characterizes the interface drag coefficient for a single particle in an infinite medium, $\mathrm{Re}_{\mathrm{s}}$ is the Reynolds number based on the particle mean diameter $d_{p}, \phi_{s}$ is the particle sphericity, $\mu$ and $\lambda$ represent dynamic and volumetric viscosities $(\mathrm{kg} / \mathrm{m}-\mathrm{s}), \theta$ stands for the granular temperature $\left(\mathrm{m}^{2} / \mathrm{s}^{2}\right)$, e represents the particle-particle restitution coefficient, $\mathrm{R}_{\mathrm{g}}$ is the ideal gas constant $(\mathrm{J} / \mathrm{kg}-\mathrm{K})$, and $\mathrm{t}$ is the time (s).

\section{Computational Domain and Initial and Boundary Conditions}

Figure 1 shows the basic computational domain employed in the simulations. The Cartesian coordinate system is applied. The geometric dimensions and initial and boundary conditions are specified in Figure 1. For the gas phase is assumed the full adherence to the wall or the no-slip boundary condition. For the solids phase is assumed a partial-slip condition in the direction tangential to the wall, in accordance with Ding and Gidaspow (1990), and the no-slip condition for the normal direction are assumed. For pressure and solids volumetric fractions a null gradient normal to the wall is assumed. Since the partial-slip condition for the solids phase was not available in the MFIX code, it was implemented. 


\section{Continuation Table 2}

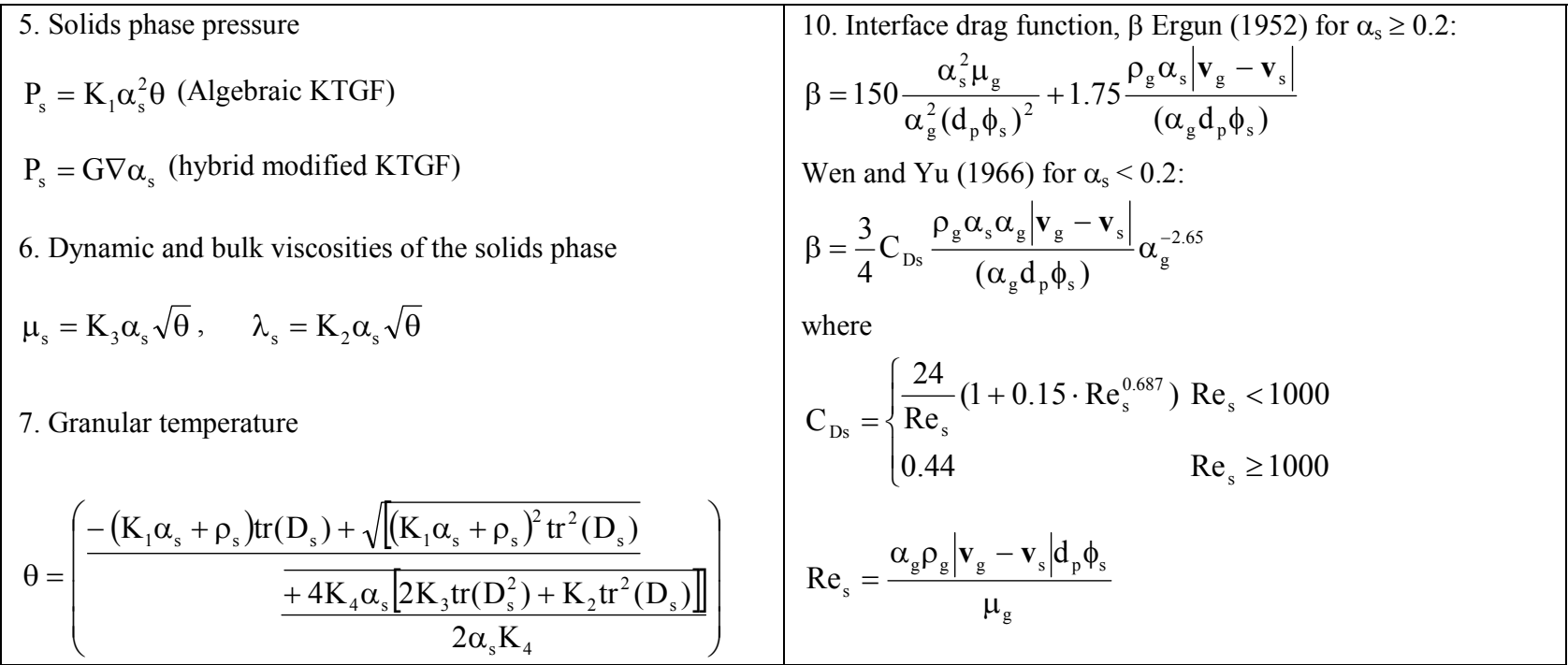

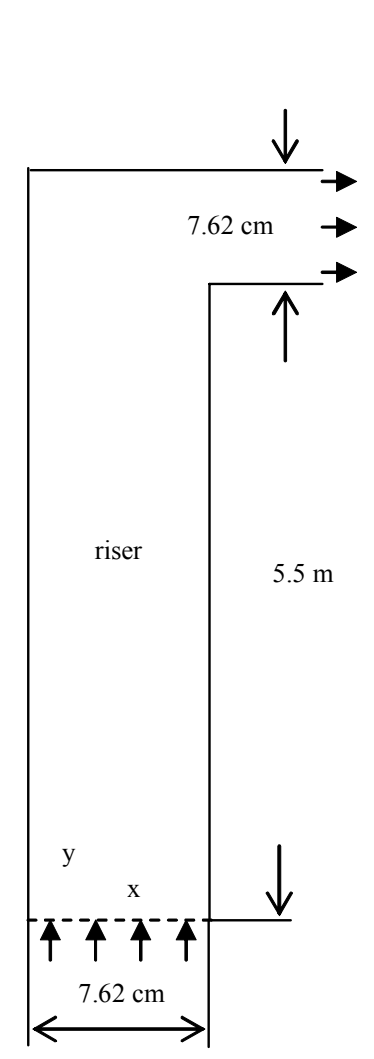

Simulation data:

Solids particle diameter: $d_{p}=520 \mu \mathrm{m}$

Solids particle density: $\rho_{\mathrm{s}}=2620 \mathrm{~kg} / \mathrm{m}^{3}$

Solids recirculation rate: $\mathrm{G}_{\mathrm{s}}=24.9 \mathrm{~kg} /\left(\mathrm{m}^{2} \mathrm{~s}\right)$

Gas phase dynamic viscosity: $\mu_{\mathrm{g}}=1.8 \times 10^{-5} \mathrm{~Pa} \mathrm{~s}$

Initial conditions:

Empty riser

$\mathrm{P}=101.325 \mathrm{kPa}$

$\mathrm{T}=300 \mathrm{~K}$

Inlet boundary conditions:

$\mathrm{v}_{\mathrm{s}}=0.386 \mathrm{~m} / \mathrm{s}$

$\mathrm{v}_{\mathrm{g}}=4.979 \mathrm{~m} / \mathrm{s}$

$\alpha_{\mathrm{s}}=0.0246$

$\mathrm{P}=120.6639 \mathrm{kPa}$

$\mathrm{T}=300 \mathrm{~K}$

Outlet boundary conditions:

Continuity condition: $\frac{\partial \mathrm{f}}{\partial \mathrm{n}}=0$

where $\mathrm{f}$ is either $\alpha_{\mathrm{g}}, \mathrm{u}_{\mathrm{g}}$, or $\mathrm{u}_{\mathrm{s}}$.

Gas phase pressure: $\mathrm{P}=117.2049 \mathrm{kPa}$

Wall boundary conditions:

Gas phase: no-slip

Solids phase: partial slip $\overrightarrow{\mathrm{v}}_{\mathrm{s}, \mathrm{w}}=-\frac{\mathrm{d}_{\mathrm{p}}}{\alpha_{\mathrm{s}}^{1 / 3}} \frac{\partial \overrightarrow{\mathrm{v}}_{\mathrm{s}}}{\partial \mathrm{n}}$

Solids fraction and pressure: null normal gradient

Computational conditions:

Numerical meshes:

Mesh 1: $(10 \times 146) 1460$ nodes: $\delta \mathrm{x}=0.762 \mathrm{~cm}, \delta \mathrm{y}=3.81 \mathrm{~cm}$

Mesh 2: $(15 \times 146) 2190$ nodes: $\delta x=0.508 \mathrm{~cm}, \delta y=3.81 \mathrm{~cm}$

Mesh 3: $(20 \times 146) 2920$ knots: $\delta x=0.381 \mathrm{~cm}, \delta y=3.81 \mathrm{~cm}$

Simulation time: $\mathrm{t}=100 \mathrm{~s}$

Figure 1: Geometry and initial and boundary conditions used in the simulations assuming Cartesian coordinates. (In accordance with Luo, 1987). 


\section{Numerical Predictions and Discussion}

Results of numerical simulation are presented for the different mathematical models, considering two different discretization schemes for the advective terms, and three different mesh sizes.The mathematical models are the traditional, the algebraic KTGF, and the hybrid modified KTGF. The discretization schemes are the first-order upwind scheme (Foup) and the second-order upwind Superbee scheme (Syamlal et al., 1993). The mesh sizes are defined in Figure 1. All the simulations presented in the paper were performed on two computers: a

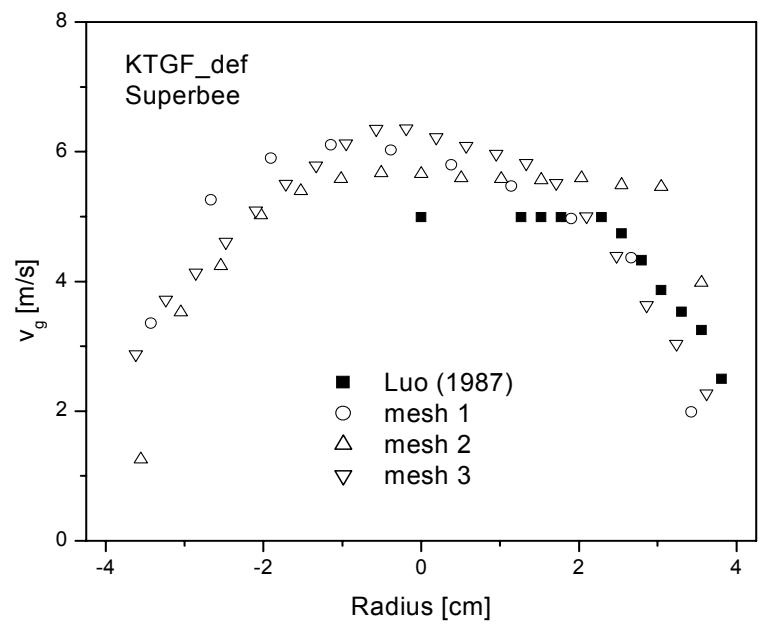

Axial velocity for the gas phase
Pentium IV with an Intel processor of $1.4 \mathrm{Ghz}$ and 512 Mbytes of Ram and an AMD-ATHLON dual computer with processors of $2.0 \mathrm{Ghz}$ and 1.5 Gbytes of RAM. The unavailability of more powerful computational resources resulted in the use coarse meshes, as explained in the following paragraphs.

The results obtained with the algebraic KTGF (KTGF_def) for the three different meshes are presented in Figure 2. The graphs show timeaveraged radial profiles of the axial velocity of both phases and the solids volumetric fraction, 3.4 meters above the riser entrance. The results are compared to Luo's experimental data (Luo, 1987).

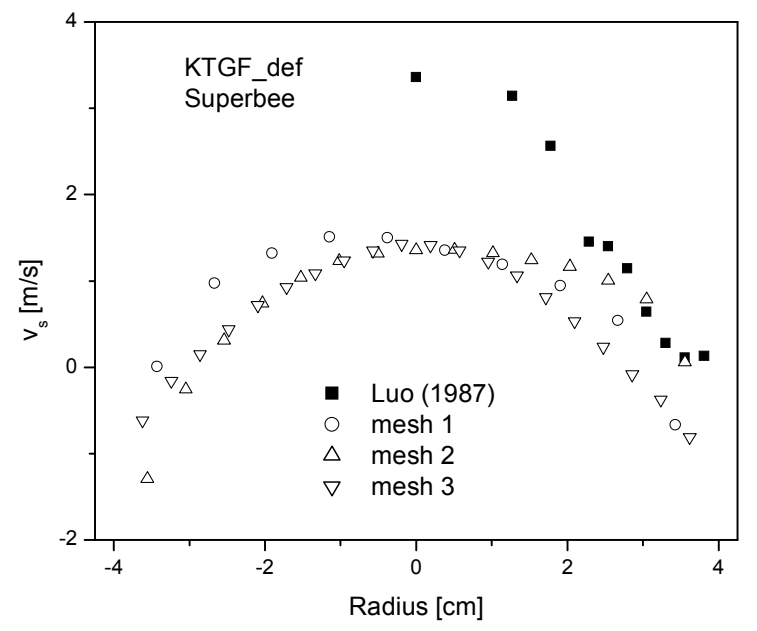

Axial velocity for the solids phase

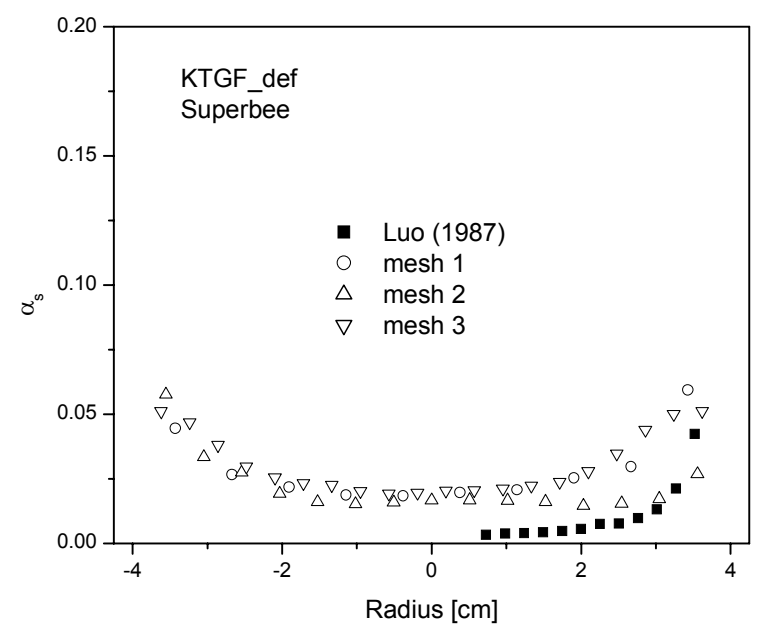

Solids phase volume fraction

Figure 2: Radial profiles for time-averaged parameters compared to Luo's experimental data, 3.4 meters above the inlet for three different meshes, Superbee advection discretization scheme and the algebraic KTGF model. 
The results for the different computational meshes show the same qualitative behavior. The quantitative predictions usually become more precise as the mesh is refined. However, there is no guarantee that the results are free from the effect of mesh size, since very coarse meshes were used. In fact, the mesh refinements used in this work, bound by computational limitations, may be far from that required for accurate meshindependent predictions. Of course, mesh should be further refined if its effect on the predictions is to be assessed. To what extent to refine, however, is not known, and a great deal of computational experimentation on this matter is still required. Regarding the comparison to Luo's experimental data, considerable deviations are observed for all parameters

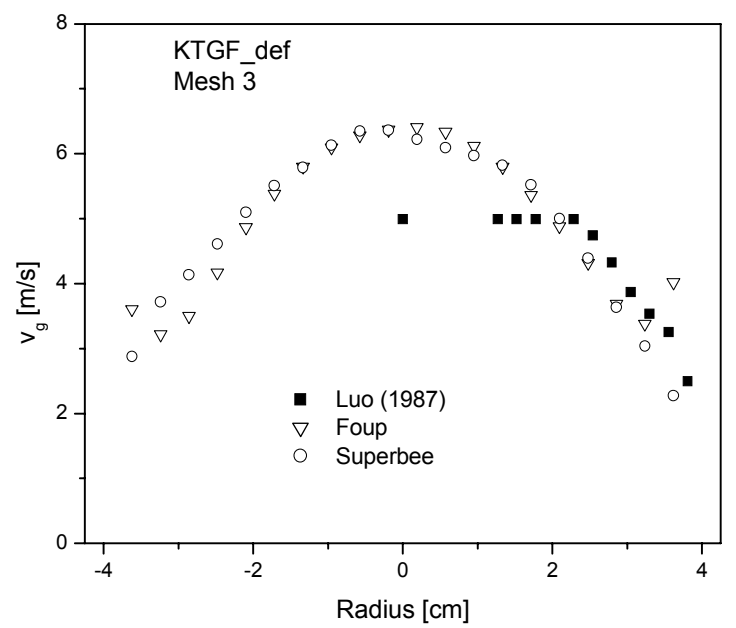

Axial velocity for the gas phase even though qualitative agreement is found. In fact, the order of magnitude of the observed quantitative deviations is quite common in CFB gas-solids flow predictions (Cabezas-Gómez, 2003). The discrepancies should be credited to both empirical difficulties of measurement and a crude stage of development of the two-fluid models applied to gas-solids flows.

Figure 3 shows comparisons of predictions using the first-order upwind (Foup) and the higher order (Superbee) discretization schemes. The algebraic KTGF and the finer $20 \times 146$ mesh were used. In comparison to Luo's experimental data, whatever the discretization scheme, the discrepancies become greater towards the center of the column for all the parameters concerned.

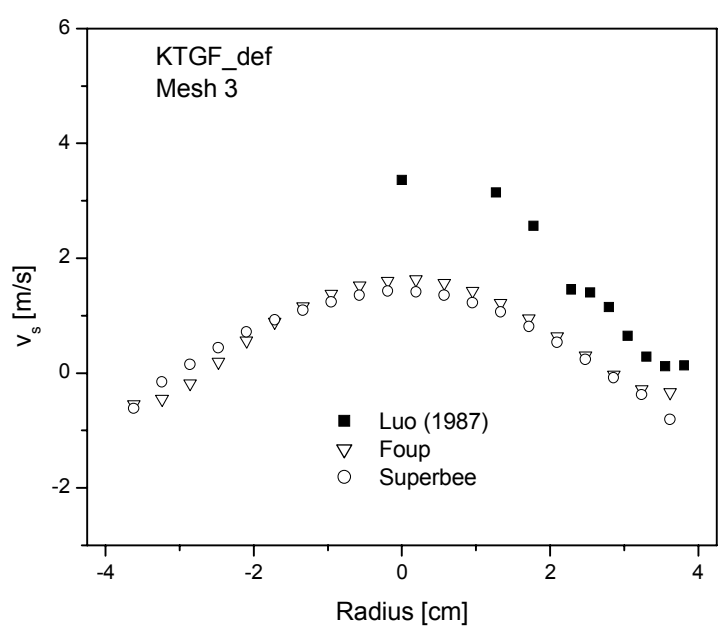

Axial velocity for the solids phase

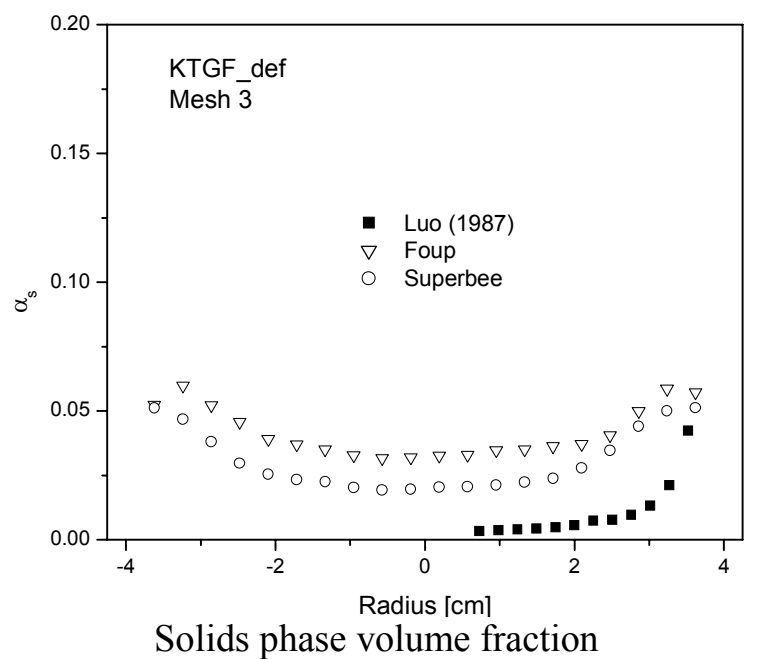

Figure 3: Radial profiles for time-averaged parameters compared to Luo's experimental data, 3.4 meters above the inlet, for Foup and Superbee advection discretization schemes, mesh 3, and the algebraic KTGF model. 
The higher order discretization scheme seems to provide better results than the first-order scheme. Nevertheless, the predictions using Superbee still deviate considerably from Luo's experimental data. While preferable for providing higher order interpolations, the Superbee scheme only slightly improves the predictions over the first-order scheme. It is clear that in the present simulations the interpolation scheme was not responsible for the observed deviations between predictions and experiment. However, it is acknowledged that the choice of a discretization procedure may be quite important for accuracy, mainly taking into account the current state of the art in computational resources, which imposes relatively coarse meshes.

The very slight improvement on the predictions as Superbee is used suggests that the numerical diffusion, which is supposed to be imposed by the first-order upwind scheme, may be not significant concerning gas-solids flows in risers. This statement is in agreement with previous conclusions of Cabezas-Gómez and Milioli (2005b). In that work an anterior version of the MFIX code was used to simulate gas-solids flows in risers, applying the firstorder upwind discretization scheme. When an inviscid solids flow was assumed, the averaged radial profiles of the axial velocity for the solids phase were very flat, indicating the absence of any diffusion-like effect, whether physical or numerical.

Figures 4 show comparisons of predictions from the algebraic KTGF (KTGF_def) and the hybrid modified (KTGF_mod) models with the predictions from the traditional model. As in the previous cases, the graphs show time-averaged radial profiles of the axial velocity of both phases and the solids volumetric fraction, 3.4 meters above the riser entrance. The higher order Superbee discretization scheme and the finer $20 \times 146$ mesh were used. Again, the results are compared to Luo's experimental data.

In all the graphs it can be seen that the KTGF_mod predictions are quite different from those of the KTGF_def and are very close to the traditional model predictions (referred to as $\mu_{\mathrm{s}}=0.5 \alpha_{\mathrm{s}}$ ). This would suggest that either in the traditional and KTGF_mod models the effect of the solids phase pressure is overestimated in comparison to the viscous effects, or else, that in the KTGF_def model it is underestimated. It seems that the second alternative is more acceptable, since the predictions of both the KTGF_mod model and the traditional procedure are closer to Luo's experimental data than those of the KTGF def model. In fact, it is not surprising that the traditional model gives better predictions than the algebraic KTGF model. The empirical correlation for the pressure of the solids phase and the empirical value of the viscosity of this phase were determined for riser flows (Luo, 1987). Otherwise, the algebraic KTGF and its correlations are based on assumptions, which are more appropriate for bubbling fluidized beds. It seems that new versions of the KTGF model are required to account for both diffusive and convective transports, which are quite significant in CFB risers. The solution of a full PDE equation for granular energy seems to be necessary, despite the tremendous additional computational effort that is required.

Recently Cabezas-Gómez and Milioli (2005a) showed the large effect that the solids pressure gradient exerts on the gas-solids flow transitory behavior in risers. The present work confirms those observations.

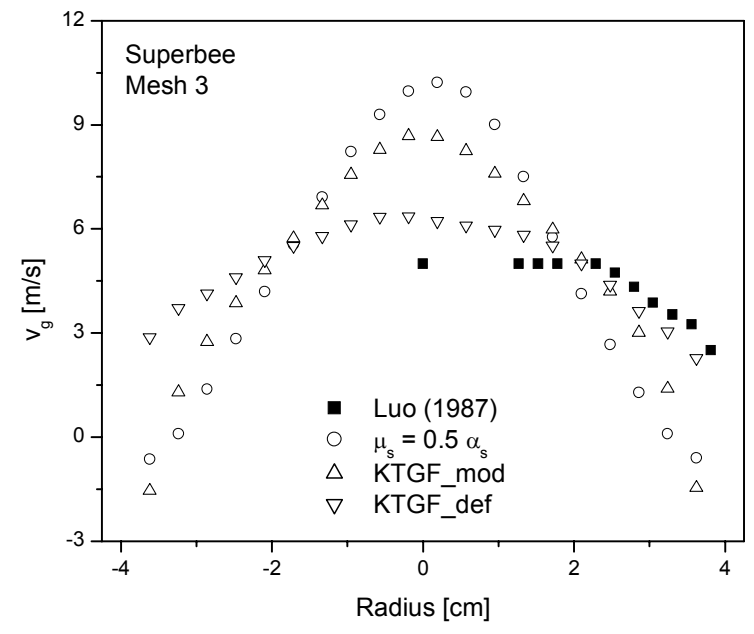

Axial velocity for the gas phase

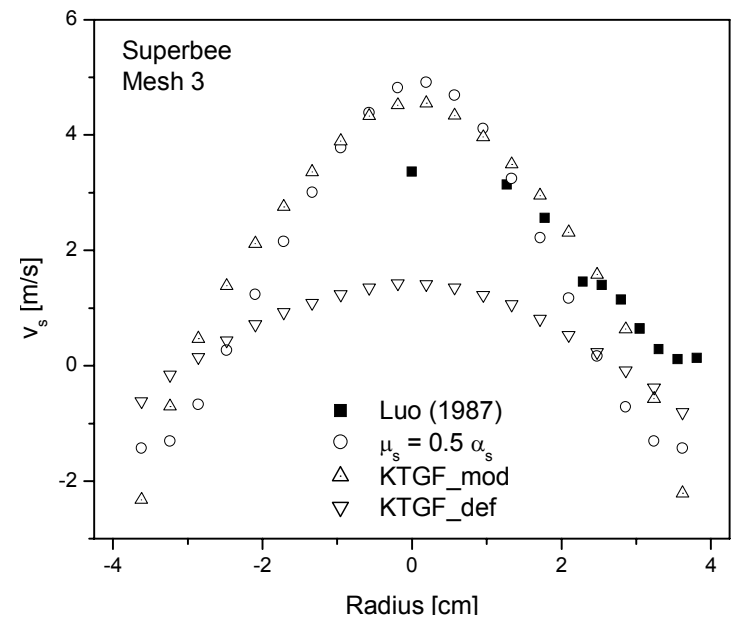

Axial velocity for the solids phase 


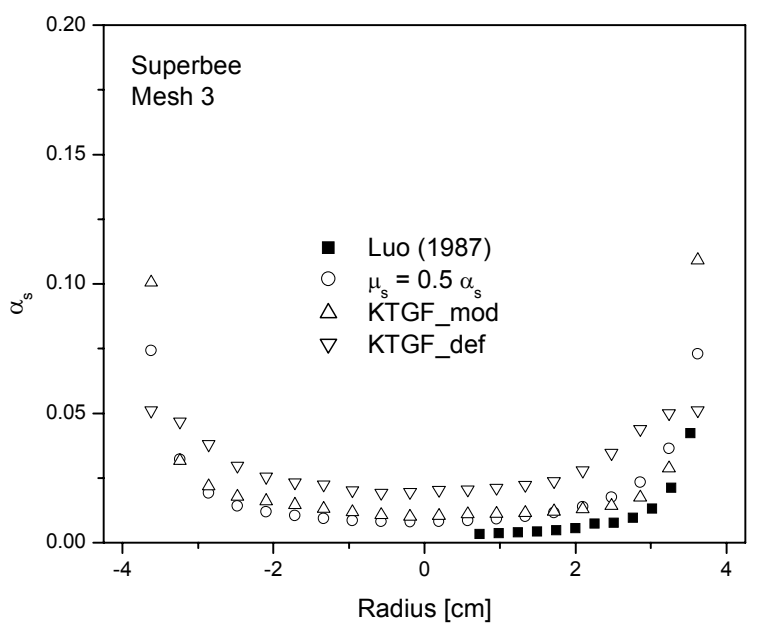

Solids phase volume fraction

Figure 4: Radial profiles for time-averaged parameters compared to Luo's experimental data, 3.4 meters above the inlet, for the algebraic KTGF, the hybrid modified KTGF and the traditional models, mesh 3, and the

Superbee advection discretization scheme.

\section{FINAL REMARKS}

The finer numerical mesh used in the simulations had twice as many cells as the coarser mesh. Even though this represents a considerable refinement, most of the predictions were very close for the two meshes. This could be taken as an indication that mesh size does not affect the predictions. However, such a conclusion is not acceptable, since even the finer mesh is still very coarse. The meshes used in this work were bound by computational limitations and are possibly very far from that required for accurate mesh-independent predictions. Further computational experimentation on this matter is clearly required.

The higher order Superbee scheme for the discretization of advection terms provided better results than the first-order Foup scheme, since predictions for the former were closer to the experimental data. Notwithstanding, the predictions by both the procedures deviated considerably from experiment. In this work the discretization scheme was clearly not responsible for the observed deviations. However, the state of the art in computational resources generally imposes coarse meshes, and the discretization scheme for advection terms may assume utmost importance. The choice should always tend towards higher order discretization schemes if accuracy is pursued.

While the higher order Superbee provided better results than the fist-order upwind Foup scheme, the predictions in fact were similar, with the exception of those associated with the solid volumetric fraction (see Figure 3), where the Superbee scheme provided better prediction results. This suggests that numerical diffusion typical of first-order upwind schemes is playing some role as far as the present simulations are concerned.

A modification of the conventional algebraic KTGF model was used by computing solids phase pressure by the empirical correlation of the traditional model. This modification brought the predictions closer to both the traditional model predictions and the experimental data. This result leads to the conclusion that the effect of the solids pressure is underestimated in the algebraic KTGF model. The algebraic KTGF is based on assumptions that are more appropriate for bubbling fluidized beds than for riser simulation. It seems that a full granular energy equation should be solved if the KTGF is to be applied to circulating fluidized bed risers.

\section{ACKNOWLEDGMENTS}

The first and second authors fully acknowledge the support respectively received from FAPESP (Fundação de Amparo à Pesquisa do Estado de São Paulo) through a postdoctoral scholarship (process 02/12038-8) and from CAPES (Coordenação de Aperfeiçoamento de Pessoal de Nível Superior) through a doctoral PICDT scholarship. The present simulations were performed with the MFIX code (freely supplied by the National Energy Technology Laboratory - www.mfix.org). The availability and support of MFIX personnel is rightly acknowledged by the authors. 


\section{NOMECLATURE}

\section{List of Symbols}

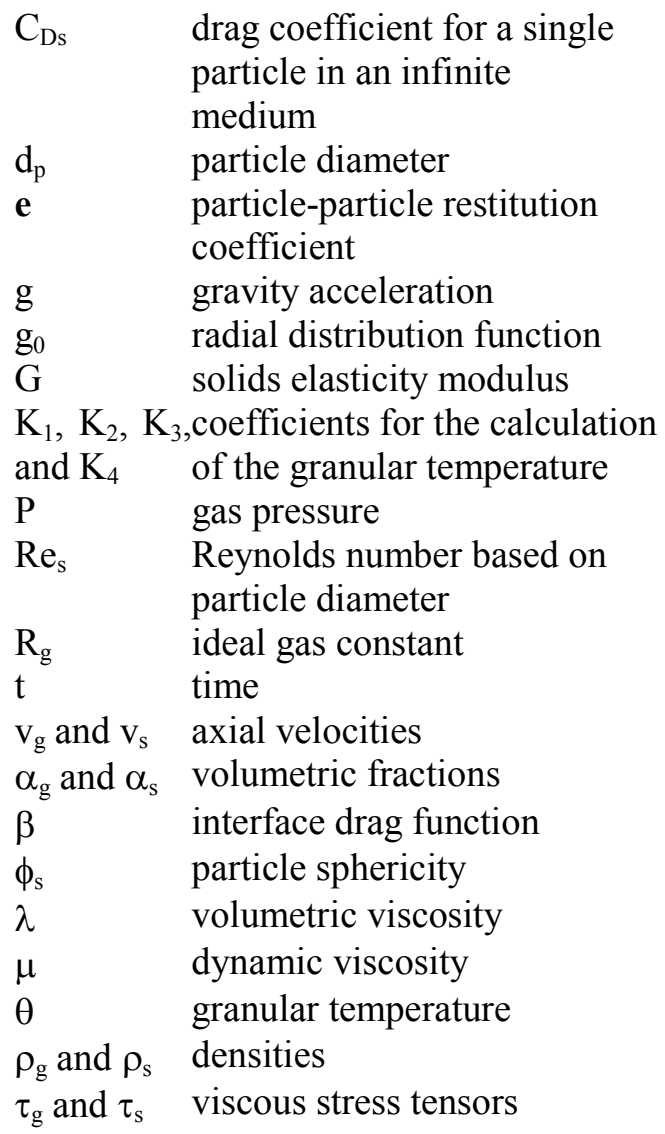

$\left(\mathrm{kg} / \mathrm{m}^{2}-\mathrm{s}\right)$

$(\mathrm{kg} / \mathrm{m}-\mathrm{s})$ $(\mathrm{kg} / \mathrm{m}-\mathrm{s})$ $\left(\mathrm{m}^{2} / \mathrm{s}^{2}\right)$ $\left(\mathrm{kg} / \mathrm{m}^{3}\right)$

\section{Subscripts}

(g) and (s) gas and solids phases

(k) gas or solids phase

\section{REFERENCES}

Boemer, A., Qi, H., Renz, U., Vasquez, S. and Boysan, F., Eulerian Computation of Fluidized Bed Hydrodynamics - A Comparison of Physical Models, In: Proc. $13^{\text {th }}$ Int. Conf. on Fluidized Bed Combustion, vol. 2, 775 (1995).

Cabezas-Gómez, L., Mathematical Modeling and Numerical Simulation of Gas-Solid Two-phase Flows in Risers of Circulating Fluidized Beds, doctoral diss., School of Engineering of São Carlos - University of São Paulo, Brazil (2003). (in Portuguese).

Cabezas-Gómez, L. and Milioli, F.E., A Parametric Study of the Gas-Solid Flow in the Riser of a Circulating Fluidized Bed Through Continuous
Eulerian Modeling, Powder Technology, 132, No. 2-3, 216 (2003).

Cabezas-Gómez, L. and Milioli, F.E., A Numerical Simulation Analysis of the Effect of the Interface Drag Function on Cluster Evolution in a CFB Riser Gas-Solid Flow, Braz. J. of Chemical Engng., 21, No. 4, 569 (2004).

Cabezas-Gómez, L. and Milioli, F.E., Collisional Solid's Pressure Impact on Numerical Results from a Traditional Two-fluid Model, Powder Technology, 149, No. 2-3, 78 (2005a).

Cabezas-Gómez, L. and Milioli, F.E., Numerical Simulation of Fluid Flow in CFB Risers - A Turbulence Analysis Approach, J. of the Braz. Soc. of Mech. Sci. \& Eng., XXVII, No. 2, 141 (2005b).

Ding, J. and Gidaspow, D., A Bubbling Model Using Kinetic Theory of Granular Flow, AIChE Journal, 36, No. 4, 523 (1990).

Ergun, S., Fluid Flow through Packed Columns, Chemical Engineering Progress, 48, No. 2, 89 (1952).

Gelderbloom, S.J., Gidaspow, D. and Lyczkowski, R.W., CFD Simulations of Bubbling/Collapsing Fluidized Beds for Three Geldart Groups, AIChE Journal, 49, No. 4, 844 (2003).

Gera, D., Syamlal, M. and O'Brien, T.J., Hydrodynamics of Particle Segregation in Fluidized Beds, Int. J. of Multiphase Flow, 30 419 (2004).

Gidaspow, D., Multiphase Flow and Fluidization: Continuum and Kinetic Theory Descriptions. Academic Press, Boston (1994).

Guenther, C. and Syamlal, M., The Effect of Numerical Diffusion on Simulation of Isolated Bubbles in a Gas-Solid Fluidized Bed, Powder Technology, 116, 142 (2001).

Huilin, L. and Gidaspow, D., Hydrodynamic Simulations of Gas-Solid Flow in a Riser, Ind. Eng. Chem. Res. 42, 2390 (2003).

Lun, C.K.K., Savage, S.B., Jeffrey, D.J. and Chepurniy, N., Kinetic Theories for Granular Flow: Inelastic Particles in Couette Flow and Slightly Inelastic Particles in a General Flow Field, Journal of Fluid Mechanics, No. 140, 223 (1984).

Luo, K.M., Dilute, Dense-Phase and Maximum Solids-Gas Transport. Ph.D. diss., Illinois Institute of Technology, Chicago (1987).

Sun, B. and Gidaspow, D., Computation of Circulating Fluidized Bed Riser Flow for the Fluidization VIII Benchmark Test, Ind. Eng. Chem. Res., 38, 787 (1999). 
Syamlal, M., Rogers, W., and O'Brien T.J., MFIX Documentation Theory Guide. U.S. Department of Energy, Morgantown Energy Technology Center, Technical Note DOE/MET-94/1004 (1993).

Tsuo, Y.P. and Gidaspow, D., Computation of Flow Patterns in Circulating Fluidized Beds, AIChE Journal, 36, No. 6, 885 (1990). van Wachem, B.G.M., Schouten, J.C., Krishna, R. and van den Bleek, C.M., Eulerian Simulations of Bubbling Behavior in Gas-Solid Fluidized Beds, Computers Chem. Engng., 22, Suppl. S299 (1998).

Wen, C.Y. and Yu, Y.H., Mechanics of Fluidization, Chemical Engineering Progress Symposium Series, 62, No. 62, 100 (1966). 